\title{
Metasynthesis of Patient Attitudes Toward Bone Densitometry
}

\author{
Aaron T. Seaman, PhD ${ }^{1,2}$, Melissa Steffen, MPH, BS , Taisha Doo, MD ${ }^{3,4}$, Heather S. Healy, MA, MLS , \\ and Samantha L. Solimeo, PhD, MPH $H^{1,2}$
}

'Department of Veterans Affairs, Center for Comprehensive Access \& Delivery Research and Evaluation (CADRE), lowa City VA Health Care System, lowa City, IA, USA; ${ }^{2}$ Department of Internal Medicine, Carver College of Medicine, University of lowa, lowa City, IA, USA; ${ }^{3} \mathrm{C}$ arver College of Medicine, University of lowa, lowa City, IA, USA; ${ }^{4}$ University of Chicago (NorthShore) Family Medicine Residency, Glenview, IL, USA; ${ }^{5}$ University of lowa Libraries, Hardin Library for the Health Sciences, University of lowa, lowa City, IA, USA.

\begin{abstract}
:
BACKGROUND: Bone densitometry (e.g., dual-energy Xray absorptiometry or "DXA") is strongly associated with osteoporosis treatment; however, rates of DXA are low. While studies have demonstrated a continued need for primary care provider education on the role of DXA in preventive care, little is known about the role of patient attitudes toward DXA. This review's purpose is to synthesize the evidence about the effects of patient perceptions and experiences of DXA on osteoporosis prevention.

METHODS: A metasynthesis was conducted of English language, peer-reviewed publications, searching relevant databases: MEDLINE, CINAHL, Web of Science Social Science Citation Index, PsycINFO, and Sociological Abstracts. Identified articles' quality was appraised using the Critical Appraisal Skills Programme (CASP) Qualitative Checklist, and an iterative process of data evaluation, integration, and synthesis was used to develop the findings.

RESULTS: Thirteen articles from ten studies were identified, composing an aggregated sample of 265 people (231 women). Participant attitudes toward screening ranged from receptive to ambivalent to concerned about results. Participants' understandings of DXA and its role in clinical care were limited. Knowledge of osteoporosis was also partial and influenced by lay sources, the media, and health care providers. Primary care providers strongly influenced participant behavior, especially if participants had a more passive approach to health care. Participants reported less concern about expected barriers of health care access and cost.

CONCLUSION: Minimal knowledge exists of patient perceptions and experiences of DXA among those who are fracture naïve: Prior research has focused primarily on secondary fracture prevention contexts. Our metasynthesis reveals patients' significant reliance, given their limited risk appraisal and knowledge, upon primary care providers in decision-making. We urge colleagues to conduct qualitative research on DXA barriers among general primary care population in order to facilitate health care delivery systems better equipped to diagnose and treat patients before their first fracture.
\end{abstract}

Electronic supplementary material The online version of this article (https://doi.org/10.1007/s11606-018-4587-3) contains supplementary material, which is available to authorized users.

Received May 18, 2018

Revised July 9, 2018

Accepted July 10, 2018

Published online July 27, 2018
KEY WORDS: Osteoporosis; Preventive care; Qualitative research; Systematic reviews.

J Gen Intern Med 33(10):1796-804

DOI: $10.1007 / \mathrm{s} 11606-018-4587-3$

(c) Society of General Internal Medicine (This is a U.S. government work and not under copyright protection in the U.S.; foreign copyright protection may apply) 2018

\section{INTRODUCTION}

Osteoporosis and its fracture-related sequelae are associated with significant societal costs from mortality, medications, emergency department visits, inpatient care, orthopedic surgery, rehabilitation, and outpatient care. ${ }^{1-3}$ In the USA, osteoporotic fractures resulted in 4.9 million hospitalizations between 2000 and 2011 among women over 55 years of age ${ }^{4}$ and, in 2005, cost an estimated 16.9 billion USD. ${ }^{3}$ Osteoporosis's individual costs are also notable: In addition to chronic pain and spinal deformation, the fear of falling causes patients considerable psychosocial distress. ${ }^{5}$ Given current global demographic trends of an aging population, ${ }^{3}$ identification of atrisk individuals, especially before they experience a fracture, presents an opportunity for significant cost savings and reduction in suffering. ${ }^{6,7}$ Early identification of those with or at risk of developing osteoporosis through bone mineral densitometry (i.e., dual-energy X-ray absorptiometry, "DXA") can lead to subsequent intervention to prevent fractures. ${ }^{8}$

Despite its considerable burdens, osteoporosis is a persistently underdiagnosed and undertreated condition, particularly among men, prompting some to decry osteoporosis care and treatment as "in crisis." "-11 DXA is underutilized, despite being a widely available, low-cost, and non-invasive diagnostic tool that requires a short office visit. ${ }^{8,} 12$ DXA underutilization is concerning because of both its critical role in prefracture assessment and its importance to clinical decisionmaking tools for treatment such as the WHO Fracture Risk Assessment Tool (FRAX). ${ }^{13}$

Interventions to improve osteoporosis identification and treatment have primarily targeted the post-fracture health care delivery system, reflecting both opportunities to leverage fractures as a teachable moment for patients with high fracture risk and a recognition of gaps in primary care provider osteoporosis knowledge. ${ }^{14-20}$ Fracture liaison services, in particular, have shown 
success in facilitating post-fracture care management. ${ }^{21-23}$ However, successful systems must include not only these services, but also primary care providers identifying at-risk patients and referring them for DXA and patients who are receptive. Recent reviews, detailing the range of qualitative work on patient experience of osteoporosis, ${ }^{24}$ its diagnosis, ${ }^{25}$ and patients' perceived health care needs ${ }^{26}$ elucidate the experience of living with osteoporosis and offer insight for, especially, post-diagnostic, post-fracture intervention. To design effective bone health care delivery systems, however, it is critical that we also understand patient decision-making processes around DXA. Thus, in the current study, we report on a qualitative metasynthesis of the research examining patient perceptions and experiences of DXA.

\section{METHODS}

We conducted a metasynthesis, which is an integrative approach to systematic reviews of qualitative research. The strength of metasynthesis lies in reviewers' ability to both analyze the empirical findings of studies and critically examine the context in which studies were produced. ${ }^{27-30} \mathrm{We}$ followed the ENTREQ protocol for conducting all steps of the review, a standard protocol for reviews of qualitative research. ${ }^{31}$ See Appendix 1 (online).

\section{Search Strategy}

The search protocol was developed in collaboration with a health sciences librarian trained in systematic review methodology $(\mathrm{HH})$ who executed the search in the following databases: MEDLINE (Ovid platform), CINAHL (EBSCO platform), Web of Science Social Science Citation Index, PsycINFO (EBSCO platform), and Sociological Abstracts (ProQuest platform). Validated, hybrid qualitative filters were applied to the MEDLINE, CINAHL, and Social Science Citation Index search strategies. ${ }^{32}$ Adjustments to the filters were made where needed to reflect current indexing. Results were limited to English language publications between January 1998 and March 2017 to identify only those studies published after the 1998 National Osteoporosis Foundation's guidelines for bone densitometry. ${ }^{33}$ Search results were updated in November 2017 prior to the final analysis. The MEDLINE search is available in Appendix 2 (online). Other database search strategies are available upon request.

A summary of the search results by database and final selection criteria is presented in Fig. 1. After deduplication by the librarian, the research team (TD, AS, SS, MS) screened articles using a two-stage process to apply criteria developed from the research question. Inclusion criteria were (1) original research or quality improvement, (2) qualitative data, (3) focus on osteopenia and/or osteoporosis AND also address bone densitometry, and (4) subjects were ages 40 and older. The age of 40 was selected as a cutoff to include only studies pertaining to older adults and to exclude studies conducted with children or young adults who likely present with different clinical risks and who may have different attitudes toward bone health. In the first stage, we divided 5145 citations among four team members (AS, MS, TD, SS), who worked in pairs to screen article titles and abstracts using the first two inclusion criteria, resulting in 171 potential articles. We reviewed the retrieved references and identified 61 additional citations for potential inclusion. In the second stage of review, three members (AS, MS, TD) reviewed 232 full text articles with all inclusion criteria. In both stages, a third person resolved disagreements and any remaining concerns were resolved by consensus among the group.

\section{Quality Appraisal}

We evaluated the quality of the included articles using the Critical Appraisal Skills Programme (CASP) Qualitative Checklist, a ten-item quality appraisal tool for qualitative research. ${ }^{34}$ Items include the appropriateness of a study's design, recruitment processes, and data collection methods, the rigor of analysis and clarity of presented findings, and ethical considerations. Three members (TD, AS, SS) independently appraised the articles, and resolved any discrepancies by consensus. See Appendix 3 (online).

\section{Analysis}

To do the integrative work of metasynthesis, we analyzed the articles iteratively, which allowed for the development and refinement of codes and themes over time. First, we (AS, MS, SS) independently read and hand-coded three articles using an inductive open coding approach. For each of the first three articles, we met as a team to compare and refine codes, resulting in a consensus-derived codebook. Upon completion of hand-coding, we imported the articles into qualitative data management software program MAXQDA 12 ${ }^{\mathrm{TM}}$ (VERBI $\mathrm{GmbH}$ ) to facilitate the remaining analysis. We (AS, MS) then coded the remaining articles. After initial coding, two authors (AS, SS) independently reviewed the coded data to develop synthetic themes. The themes were reviewed and integrated through a consensus process, resulting in four overarching insights derived from the studies, summarized below.

\section{RESULTS}

Thirteen articles reporting on ten studies met the inclusion criteria and were analyzed. See Table 1 for a summary of study characteristics. Two research groups from Canada accounted for 8 of the articles. ${ }^{35-42}$ The remaining articles were from teams located in the USA (3), ${ }^{43-45}$ UK (1), ${ }^{46}$ and Denmark (1), ${ }^{47}$ and all articles were published within the last 15 years. Two studies also interviewed provider participants; ${ }^{44,46}$ for the purposes of this analysis, only patient data were included. All articles were appraised at moderate to high quality, ranging in scores on the CASP qualitative quality appraisal tool from 7 to 10. See Appendix 3 (online). 


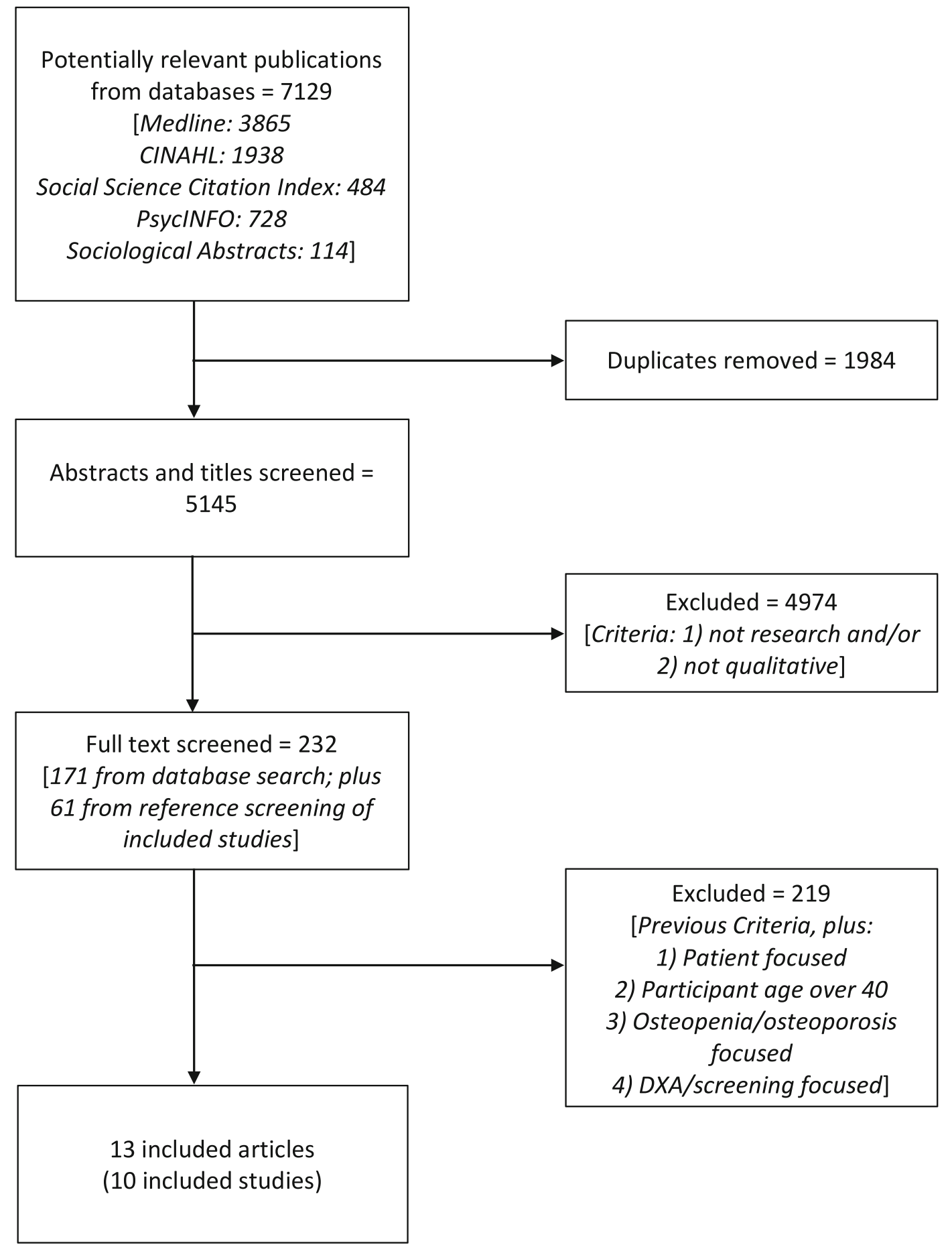

Fig. 1 Search process and screening results. The figure illustrates the search and screening process that resulted in the metasynthesis's included articles, including the number of articles and exclusion criteria at each step

Across the ten studies, a total of 265 people (231 women and 34 men) participated. See Table 2. Participants were recruited through clinical settings, ${ }^{35-37,40-42,44,45}$ as part of larger randomized controlled study trials, ${ }^{46,}, 47$ from an osteoporosis patient group, ${ }^{38,39}$ or the community. ${ }^{43}$ The majority of studies recruited only women. ${ }^{40-44,46,47}$ At least 104 participants, from six studies with 159 total participants, had been diagnosed with osteoporosis. ${ }^{36,38,39,42,43,45,47}$ Rates of DXA were reported in half of the studies. ${ }^{35-39,43,44,46,47}$ Most studies reported pharmacotherapy or mineral supplement use, ${ }^{35-39,42-46}$ with 88 out of 193 participants reporting current or prior use.

Studies differed regarding their focus on patient attitudes toward DXA, bone health, and care delivery, with some focused on women's experiences of fractures and risk perceptions, ${ }^{40-42}$ others more broadly investigating understanding of 
Table 1 Research Design Characteristics

\begin{tabular}{|c|c|c|c|c|}
\hline Author & Year & Country & Design & $\begin{array}{l}\text { Recruitment } \\
\text { setting }\end{array}$ \\
\hline Beaton et al. $^{\text {a }}$ & 2012 & Canada & Observational & Clinical settin \\
\hline Edwards et al. & 2006 & USA & $\begin{array}{l}\text { Mixed methods, } \\
\text { prospective study, } \\
\text { and focus groups }\end{array}$ & Community \\
\hline Emmett et al. & 2012 & UK & $\begin{array}{l}\text { Observational sub- } \\
\text { study of Screening } \\
\text { of Older Women } \\
\text { for Prevention of } \\
\text { Fracture (SCOOP) }\end{array}$ & $\begin{array}{l}\text { Sub-study of } \\
\text { RCT }\end{array}$ \\
\hline Feldstein et al. ${ }^{a}$ & 2008 & USA & Observational & Clinical settin \\
\hline
\end{tabular}

Primary objective

Interview

type

"The objective of this study was to interview

patients at risk for an additional [osteoporosis]-

related fragility fracture and to understand their

experiences of [osteoporosis] awareness, diagnosis,

testing, and treatment within the context of a

coordinator-based system in an orthopedic fracture

clinic setting. We also sought to identify factors

associated with the successful uptake of testing and treatment recommendations."

"To explore women's perceived barriers to seek medical care for osteoporosis following a MTF [minimal trauma fracture]"

"The qualitative study reported here explored the acceptability of the screening process from the perspective of women and GPs with the aim of informing future implementation."

\section{Focus}

group $(5)^{\mathrm{d}}$

"The focus groups and interviews elicited barriers and facilitators to screening and treating osteoporosis, perceived utility of the outreach program, and overall advice on how to improve screening and treatment of osteoporosis. Our study's immediate goal was to use the findings reported here to improve the outreach program in the future, with the long-term goal of improving osteoporosis care and fracture prevention."

Meadows and $2003 \quad$ Canada Observational Clinical setting
Mrkonjic

Meadows et al. ${ }^{\mathrm{b}} 2004$ Canada Observational Clinical setting

Meadows et al. ${ }^{\text {b }} 2005$ Canada Observational Clinical setting

Rothmann et al. 2014 Denmark Qualitative sub-study of Risk-stratified Osteoporosis Strategy Evaluation (ROSE)

Sale et al. ${ }^{\mathrm{a}} \quad 2010 \quad$ Canada $\quad$ Observational Clinical setting

Sale et al. 2014a Canada Observational Clinical setting

Sale et al. ${ }^{\mathrm{c}} \quad 2014 \mathrm{~b} \quad$ Canada $\quad$ Observational National Osteoporosis Patient Group

\begin{tabular}{|c|c|c|c|c|}
\hline Sale et al. ${ }^{\mathrm{c}}$ & 2015 & Canada & Observational & $\begin{array}{l}\text { National } \\
\text { Osteoporosis } \\
\text { Patient Group }\end{array}$ \\
\hline Solimeo et al. & 2011 & USA & Observational & Clinical setting \\
\hline
\end{tabular}

\footnotetext{
${ }_{a, b, c}$ Articles from the same studies
}

${ }^{d}$ Number of focus groups conducted
"This manuscript presents data from a pilot study that explored the questions, 'What are women's experiences of fractures at midlife?' and 'What connection is made between those fractures and bone health by women and their physicians?"'

"This descriptive and exploratory study is part of an on-going program of research focused on midlife women and bone health." "In this article we highlight the perceptions of midlife women regarding their fracture experiences as it affects their own risk of future compromised bone health."

"We report here on a study of women's experiences of fractures and present data in which women reflect on their perceived fracture risk after having suffered a lowenergy fracture at midlife (i.e., 40 years and beyond)."

"The aim of the present qualitative study was to explore women's perspectives and experiences in relation to an osteoporosis screening trial (riskstratified osteoporosis strategy evaluation, ROSE), with a specific focus on acceptance and psychological consequences of screening."

"The purpose of this study was to examine fracture patients' understanding of [osteoporosis] and [osteoporosis] care after being screened for [osteoporosis] in a fracture clinic."

"The purpose of the current qualitative study was to examine patients' experiences and actions regarding [osteoporosis] investigation and treatment after they were screened through the [Fracture Clinic

Screening Program]. Specifically, we were interested in the potential barriers to post-fracture secondary prevention experienced by, or influencing, patients before and after fracture risk assessment."

"To narrow existing investigation and treatment gaps in bone health, the purpose of our study was to examine the experiences and behaviours with bone health management post-fracture among members of an existing national [osteoporosis] patient group." "The purpose of our study was to determine how members of a national [osteoporosis] patient group perceived the messages received from various healthcare providers regarding bone health following a fracture."

"This study demonstrates the variance and commonalities found among men's experience [of osteoporosis] and raises the clinical implications of patient beliefs."
Focus group (6)

Semistructured

Semistructured

In-depth

Focus group (4)

In-depth

Focus group (8)

Semi-

structured

Focus group (5)

Interviews (2; 1 st at 6 months, 2nd at 18 months)

Interviews

Interviews

Semistructured 
Table 2 Participant Characteristics

\begin{tabular}{|c|c|c|c|c|c|c|c|}
\hline$\overline{\text { Study }}$ & $\mathbf{N}$ & $\begin{array}{l}\text { Number } \\
\text { of men } \\
n(\%)\end{array}$ & Ages & $\begin{array}{l}\text { Prior } \\
\text { fracture } \\
n(\%)\end{array}$ & $\begin{array}{l}\text { DXA } \\
n(\%)\end{array}$ & $\begin{array}{l}\text { Pharmacotherapy or mineral } \\
\text { supplementation } \\
n(\%)\end{array}$ & $\begin{array}{l}\text { Osteoporosis } \\
\text { diagnosis } \\
n(\%)\end{array}$ \\
\hline Beaton et al. ${ }^{\mathrm{a}}$ & 24 & $6(25)$ & $47-80$ & $24(100)$ & $22(92)$ & $20(83)$ & $\mathrm{NR}^{\mathrm{d}}$ \\
\hline Edwards et al. & 29 & 0 & $\geq 40$ & $29(100)$ & $16(55)$ & $9(31)$ & $26(90)$ \\
\hline Emmett et al. & 30 & 0 & $\geq 70$ & $8(27)$ & $30(100)$ & $7(23)$ & NR \\
\hline Feldstein et al. & 10 & 0 & $\geq 67$ & $10(100)$ & 0 & 0 & NR \\
\hline $\begin{array}{l}\text { Meadows and } \\
\text { Mrkonjic }\end{array}$ & 19 & 0 & $\overline{4} 0-65$ & $19(100)$ & NR & NR & NR \\
\hline Meadows et al. (2004) ${ }^{\mathrm{b}}$ & 24 & 0 & $\geq 40$ & $24(100)$ & NR & $\sim 12(\sim 50)^{\mathrm{e}}$ & $\sim 12(\sim 50)$ \\
\hline Meadows et al. $(2005)^{\mathrm{b}}$ & 22 & 0 & $\bar{N} R^{f}$ & $22(100)$ & NR & NR & NR \\
\hline Rothmann & 31 & 0 & $65-80$ & NR & NR & NR & $6(19)$ \\
\hline Sale et al. $(2010)^{\mathrm{a}}$ & 24 & $6(25)$ & $47-80$ & $24(100)$ & $21(88)$ & $\begin{array}{l}9(38, \text { bisphosphonate }) \\
1(4, \text { hormone replacement } \\
\text { therapy }) \\
16(67, \text { calcium and vitamin } D) \\
2(8, \text { calcium }) \\
1(4, \text { vitamin } D)\end{array}$ & $\sim 12(\sim 50)$ \\
\hline Sale et al. (2014a) & 25 & $3(12)$ & $\geq 50$ & $25(100)$ & $18(72)$ & $\sim 4(\sim 16)$ & NR \\
\hline Sale et al. $(2014 b)^{c}$ & 28 & $2(7)$ & $\overline{5} 1-89$ & $28(100)$ & $28(100)$ & $19(68)$ & $25(89)$ \\
\hline Sale et al. $(2015)^{\mathrm{c}}$ & 28 & $2(7)$ & $51-89$ & $28(100)$ & $28(100)$ & $19(68)$ & NR \\
\hline Solimeo et al. & 23 & $23(100)$ & $\geq 50$ & $13(57)$ & NR & $17(74)$ & $23(100)$ \\
\hline
\end{tabular}

${ }^{a, b, c}$ Articles from the same studies

${ }^{d}$ Not reported

"Only estimates reported

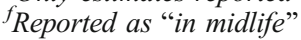

osteoporosis and osteoporosis care, ${ }^{35-37,39}$ and one examining men's experiences of osteoporosis. ${ }^{45}$ One article's analysis examined patient health care-seeking behaviors, categorizing them as more or less effective "consumers" of health care, ${ }^{38}$ and two framed their analysis in terms of barriers and facilitators to bone health care. ${ }^{43,44}$ The majority of authors reported on patient perspectives of bone health in terms of risk, screening, diagnosis, and treatment; two focused specifically on screening. ${ }^{46,47}$ Below, we synthesize the attitudes of participants across studies toward DXA, as well as participants' osteoporosis knowledge, and perceptions of providers and health care systems. We then summarize the authors' analyses, situating them within current theories of health behavior.

\section{Attitudes Towards and Understanding of DXA}

Participants articulated a range of attitudes toward DXA, variously describing DXA as an opportunity to "establish a baseline" 41 or to reassure themselves about their bone health; ${ }^{47}$ a means to explore concerns "about their bones" 38 or to seek diagnosis; $;{ }^{45}$ or a preventive measure

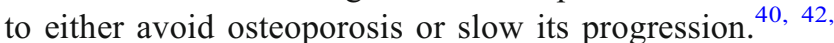
46, 47 Others noted concerns about DXA pertaining to (1) a perceived mismatch between their sense of well-being and DXA results indicating bone disease ${ }^{41,42,46,47}$ and (2) the stigma and the mental health impact of being labeled as a person at "high risk" or "with osteoporosis." ${ }^{\text {" }}$ Participants in three studies, ${ }^{35,40,42}$ while not averse to DXA, discussed not having considered it, indicating that they relied upon their provider's opinion, as one person commented: "If my doctor thought it was something I should have done, I would do it." $35,40,42$ Additionally, participants reported confusion about DXA's purpose, ${ }^{36}$ the nature of the ex$\mathrm{am},{ }^{35,36,46}$ and interpretation of results. ${ }^{35,37,41}$

\section{Role of Osteoporosis Knowledge in Bone Health Care}

Participants expressed uncertainty regarding bone health and the etiology of osteoporosis, ${ }^{36,39,43,45}$ and some confused osteoporosis with osteoarthritis. ${ }^{43,}{ }^{44}$ Participants characterized osteoporosis as an often-inevitable condition of older women ${ }^{35}, 36,40,42-44,46,47$ and in one study, explicitly downplayed its severity. ${ }^{47}$ Interviewees perceived osteoporosis to be a relatively minor health concern compared to cancer or stroke, with one commenting that "[osteoporosis] isn't something you die of." Interviewees reported "healthy" lifestyle behaviors, primarily diet and exercise, as factors that would preclude them from bone health risk, ${ }^{42,} 47$ and many did not associate osteoporosis with fractures. ${ }^{35,36,39-43}$ Those with fracture history described fractures as isolated, circumstantial events, and attributed falls to idiosyncrasies (e.g., poor footwear, bad vision, or distractedness) or environmental factors (e.g., weather, uneven sidewalks, or stairs). ${ }^{35}, 36,40,42,43$ The few participants who connected osteoporosis with fracture risk did not extend this to their own health history. ${ }^{42}$ Information needs were addressed by some participants. Whereas some participants actively sought information from health care providers, ${ }^{35-41,43-45}$ family, ${ }^{35,} 42,47$ and media, ${ }^{40,} 44$ others waited for providers to provide them with the necessary information. ${ }^{35,37,38,42}$ In four studies, participants described receiving no or inadequate information from health care providers. ${ }^{37,39,43,47}$ 


\section{Health Care Provider Role in DXA Utilization}

Participants' descriptions of their health care encounters positioned primary care providers as having significant impact on patient knowledge, risk appraisal, and willingness to pursue DXA. Providers were seen as having the potential to offer either clarifying insight into participants' bone health or information that left participants confused. ${ }^{35-46}$ Participants noted the potential of primary care providers, ${ }^{35,} 39-42$ specialists, ${ }^{38-41}$ nurses, ${ }^{39}$ screening coordinators, ${ }^{35}$ and other providers ${ }^{39}$ to facilitate or discourage densitometry. Provider guidance was associated with bone health care in several studies. ${ }^{35,} 37-39,42,44,45$ In some cases, this guidance activated patients toward DXA, ${ }^{35,39,44}$ as one recalled, "I just went [for a BMD] because she [physician] suggested I should go." Yet, in nine articles, participants recalled provider guidance as a potential deterrent to DXA. ${ }^{35,37-43,45}$ Examples of deterrence included providers failing to discuss bone health or downplaying the necessity of DXA, ${ }^{35,37-39,41-43}$ instances where patients advocated for information themselves due to their perception that providers were pressed for time, ${ }^{35,} 37-39,42,45$ inconsistent guidance across providers, ${ }^{39}$ or encounters in which interviewees reported receiving poor bone health information. For example, in one study, men attributed delayed screening, diagnosis, and treatment to provider misconceptions. ${ }^{45}$ Participants in another study described providers assessing bone health and dissuading them from DXA using characteristics such as a patient's weight, race, height, or age. ${ }^{37}$

\section{Health Care Delivery System's Role in DXA Utilization}

Reflecting our selection criteria, none of the included studies focused on health care system influence. However, participants in a few studies mentioned access, cost, and available time as factors potentially affecting DXA use. ${ }^{35,38,44,46}$ Only in one article did respondents cite geographic access to DXA facilities to be potentially problematic for patients. ${ }^{46}$ In another, participants described "ease of access" as a facilitator to DXA, although authors noted that this was "likely because most of the study participants lived in a large urban area where many BMD [bone mineral density] testing clinics were available." ${ }^{35}$ Although the authors of four articles reported participant responses concerning cost, the only discussion of cost as a potential barrier was in regard to pharmacological treatment. ${ }^{35 \text {, }}$ ${ }^{43-45}$ Participants did not express concern about insurance coverage for DXA, although the majority of studies were conducted in countries with national health insurance. ${ }^{35-42,46,47}$

\section{Synthesizing Patient Knowledge, Attitudes, and Behavior}

In several analyses, authors drew upon existing models of health behavior to contextualize their findings, highlighting how participants' understandings of bone health motivated their actions, ${ }^{35,} 36,40,43,45,47$ and, in one article, proposed their own model of patient behavior that moves from awareness to potential action in the context of hip fracture. ${ }^{35}$ Authors connected participants' osteoporosis knowledge and appraisal to information-seeking tendencies, dichotomizing participants into active and passive information seekers. ${ }^{35,38,40,42}$ Sale and colleagues discussed information-seeking behaviors as one element of a broader "effective consumer" patient. ${ }^{38}$ Across articles, patients who were active in seeking information were more likely to pursue screening and treatment, even without provider recommendations. Authors also reflected on the role of ageism and gender bias in participants' bone health beliefs. Edwards and colleagues noted that "age bias plays a role in patients down-playing or denying osteoporosis prevention." 43 This ageism, an individual's perceived mismatch between their chronological age and their social age, was linked to inaccurate assessment of fracture risk, ${ }^{41}$ a reluctance to taking medicines ${ }^{47}$ a reluctance to curtail activities or engage in fallavoidance strategies, ${ }^{47}$ normalization of osteoporosis as an unremarkable aspect of aging, ${ }^{40}$ and the apparent disconnection between living a self-described "healthy lifestyle" which precluded the possibility of also having poor bone health. ${ }^{40,42}$ Authors also identified the influence of participants' perceptions of osteoporosis as a condition particular to women, noting how a lack of information on osteoporosis among men affected how they understood the condition and its presentation in their own bodies ${ }^{45}$ and suggesting that screening and treatment guidelines should revisit gendered inclusion criteria. ${ }^{46}$

\section{DISCUSSION}

We undertook this metasynthesis to evaluate what is known about patient views on DXA as a step toward designing bone health care delivery systems that can identify patients with suspected osteoporosis before they sustain a life-changing fracture. DXA is considered the "gold standard" for clinical assessment of bone health, diagnosis of osteoporosis, and monitoring of fracture risk and treatment effects, but it remains underutilized across the spectrum of osteoporosis care. ${ }^{12}$

Qualitative research on patient attitudes toward DXA is scant and most often pertains to the context of secondary fracture prevention. Existing evidence identified in this review suggests patients' beliefs regarding the role of DXA, osteoporosis severity, and the age and gender norms embedded in those beliefs can inform efforts to improve bone health care messaging and delivery. Given a lack of clear provider ownership of osteoporosis, ${ }^{16,17,48}$ our findings reinforce the role primary care providers can play in informing patients and engaging them in appropriate decision-making about their bone health.

\section{The Role of Primary Care in Engaging Patient Use of DXA}

The findings of this review emphasize the centrality of primary care in the identification and treatment of osteoporosis. 
After a fracture, patients largely relied upon the recommendations of their health care providers, and as other studies have found, ${ }^{15,49,50}$ the reviewed studies reveal that significant knowledge gaps remain among this workforce. Patients reported that providers downplayed the importance of DXA and patients' concerns about their bone health. ${ }^{35}$ Indeed, the coincidence of social norms which define proper patient behavior as being acquiescent to provider recommendations for tests and medications, with an underlying confidence in health care providers and systems to identify medically necessary diagnostic techniques at the appropriate time, means that the likelihood of receiving a DXA is significantly dependent upon whether their health care provider clearly articulates both the relationship between fracture and bone health and the importance of monitoring bone density. While patients may also experience transportation, cost, and time barriers to DXA, participants reported the facilitative role of nurses and screening coordinators, when available, in accessing DXA.

These findings reinforce the importance of ongoing efforts to improve health care delivery systems. Given the modest effects of provider-based interventions, ${ }^{51}$ however, this review underscores the need for strategic interventions to also target patients. Regardless of primary care expertise, practitioners cannot compel patients to report for DXA or initiate pharmacotherapy if patients do not attribute fracture to osteoporosis or consider osteoporosis to be a significant health concern, a phenomenon noted by others. ${ }^{52,53}$ While participants in these studies on the whole were receptive to DXA and many had undergone post-fracture imaging, they demonstrated limited knowledge of DXA and its role in bone health management. Poor patient knowledge is compounded by reported inconsistent provider messaging and misinformation, reinforcing a point made by others regarding the importance of clinical ownership of bone health care. ${ }^{54}$ Participant descriptions of osteoporosis revealed significant age and gender biases. These perceptions threaten willingness to engage in bone health care by decreasing patient assessment of personal risk and contributing to the stigma of a potential diagnosis.

\section{Strengths and Limitations}

As in any metasynthesis, the potential exists that we have missed useful research in our search, especially reports which appear in non-peer-reviewed publications or are indexed elsewhere. In addition, the studies that we reviewed had certain limiting factors. The majority of studies were from countries with national health care systems, whose barriers may differ from what is experienced by patients in other settings. Also, few data were reported on barriers experienced by men, and their barriers likely differ from those faced by women. Moreover, despite increasing attention to the "crisis" in osteoporosis care, we were unable to identify research to directly inform the larger discussion of how to improve DXA utilization outside of the context of post-fracture care. At the same time, this apparent limitation is also a study strength, as the metasynthesis identifies a bias in the field. Published qualitative research emphasizes the role of DXA in secondary fracture prevention, eliding the screening and monitoring component as solely a means to treatment rather than as a critical component of bone health care in its own right. Thus, while the metasynthesis in some ways echoes others' calls for attention to primary care provider and patient knowledge gaps, the metasynthesis uniquely highlights the need for future research into patients' attitudes toward DXA in primary fracture prevention.

\section{Broadening the Research Agenda to Include Primary Fracture Prevention}

Our metasynthesis reveals the need for research focused on primary fracture prevention through early identification and intervention. Secondary fracture prevention was the explicit research context for seven studies accounting for ten articles included here. ${ }^{35-44}$ In part, this may be an artifact of study recruitment and access to participants. Recruitment occurred primarily through specialty clinics, ${ }^{35-37,40-42,44,45}$ as part of randomized clinical trials to improve osteoporosis care, ${ }^{46,47}$ or through an osteoporosis patient group. ${ }^{38,}{ }^{39}$ Studies also had high rates of DXA (78\% among those reporting), osteoporosis diagnoses (65\% among those reporting), and treatment use (45\% among those reporting). Participants such as these might be able to retrospectively evaluate barriers to initial screening or speculate on potential barriers others might face. However, they are not representative of fracture-naïve patients and their experiences, attitudes, and behaviors.

\section{CONCLUSION}

Interventions to address the crisis in bone health care have largely focused on secondary fracture prevention, likely due in part to the ability to leverage fracture as a teachable moment to activate patients and providers in an effort to close the treatment gap. While these measures help to prevent subsequent fractures, to successfully address the care crisis, primary fracture prevention measures in primary care settings using DXA to identify at-risk patients before fracture are warranted. In parallel, we advocate for research examining patient experiences of and barriers to DXA beyond the secondary fracture prevention context to illuminate the gaps among risk assessment, knowledge, and provider interaction that lead to underutilized bone health care.

Acknowledgments: This material is based upon work supported in part by the Department of Veterans Affairs, Veterans Health Administration, Office of Research and Development. The results of this research were presented by Dr. Solimeo at the Society for Applied Anthropology 2018 Annual Meeting in a presentation entitled, "Understanding the Crisis in Osteoporosis Care through Metasynthesis."

Corresponding Author: Aaron T. Seaman, PhD; Department of Internal Medicine, Carver College of Medicine, University of Iowa, 200 Hawkins Drive, Iowa City, IA, USA (e-mail: aaron-seaman@uiowa.edu). 
Funding Information Dr. Solimeo received support from the Center for Comprehensive Access and Delivery Research and Evaluation, Department of Veterans Affairs, Iowa City VA Health Care System, Iowa City, IA (Award No. CIN 13-412) and VA Health Services Research and Development (Award No. CDA 13-272).

\section{Compliance with Ethical Standards:}

Conflict of Interest: The authors declare that they do not have a conflict of interest.

Disclaimer: The views expressed in this article are those of the authors and do not necessarily reflect the position or policy of the Department of Veterans Affairs or the US government.

\section{REFERENCES}

1. Budhia S, Mikyas $\mathbf{Y}$, Tang $\mathbf{M}$, Badamgarav E. Osteoporotic fractures: systematic review of US healthcare costs and resource utilization. Pharmacoeconomics. 2012;30(2):147-170.

2. Becker DJ, Yun H, Kilgore ML, et al. Health services utilization after fractures: evidence from Medicare. J Gerontol A Biol Sci Med Sci 2010;65A(9):1012-1020.

3. Burge R, Dawson-Hughes B, Solomon DH, Wong JB, King A, Tosteson A. Incidence and economic burden of osteoporosis-related fractures in the United States, 2005-2025. J Bone Miner Res. Mar 2007;22(3):465-475.

4. Singer A, Exuzides A, Spangler L, et al. Burden of Illness for osteoporotic fractures compared with other serious diseases among postmenopausal women in the United States. Mayo Clin Proc. 2015;90(1):53-62.

5. Gold DT. The nonskeletal consequences of osteoporotic fractures. Psychologic and social outcomes. Rheum Dis Clin North Am. 2001;27(1):255-262.

6. Dy CJ, LaMont LE, Ton QV, Lane JM. Sex and gender considerations in male patients with osteoporosis. Clin Orthop Relat Res. 2011;469(7):1906-1912.

7. Brenneman SK, Yurgin N, Fan Y. Cost and management of males with closed fractures. Osteoporosis Int. 2013;24(3):825-833.

8. Tucci JR. Importance of early diagnosis and treatment of osteoporosis to prevent fractures. Am J Manag Care. 2006;12(7 Suppl):S181-S190.

9. Binkley N, Blank RD, Leslie WD, Lewiecki EM, Eisman JA, Bilezikian JP. Osteoporosis in crisis: it's time to focus on fracture. J Bone Miner Res. 2017;32(7): 1391-1394.

10. Khosla S, Cauley JA, Compston J, et al. Addressing the crisis in the treatment of osteoporosis: a path forward. J Bone Miner Res. 2017;32(3):424-430.

11. Khosla S, Shane E. A crisis in the treatment of osteoporosis. J Bone Miner Res. 2016;31(8):1485-1487.

12. Lewiecki EM, Laster AJ, Miller PD, Bilezikian JP. More bone density testing is needed, not less. J Bone Miner Res. 2012;27(4):739-742.

13. Siris ES, Adler R, Bilezikian J, et al. The clinical diagnosis of osteoporosis: a position statement from the National Bone Health Alliance Working Group. Osteoporos Int. 2014;25(5):1439-1443.

14. Morse LR, Giangregorio L, Battaglino RA, et al. VA-based survey of osteoporosis management in spinal cord injury. PM R. 2009; 1(3):240-244.

15. Giangregorio L, Fisher P, Papaioannou A, Adachi JD. Osteoporosis knowledge and information needs in healthcare professionals caring for patients with fragility fractures. Orthop Nurs. 2007;26(1):27-35.

16. Simonelli C, Killeen K, Mehle S, Swanson L. Barriers to osteoporosis identification and treatment among primary care physicians and orthopedic surgeons. Mayo Clin Proc. 2002;77(4):334-338.

17. Skedros JG, Holyoak JD, Pitts TC. Knowledge and opinions of orthopaedic surgeons concerning medical evaluation and treatment of patients with osteoporotic fracture. J Bone Joint Surg Am. 2006;88A(1):18-24.

18. Solomon DH, Connelly MT, Rosen CJ, et al. Factors related to the use of bone densitometry: survey responses of 494 primary care physicians in New England. Osteoporos Int. 2003;14(2):123-129.

19. McNamara M, Paik J, Beaton C, Fang M. Male osteoporosis knowledge among Veterans and their providers. Fed Pract. 2009;26(9):28-36.

20. Nelson RE, Nebeker JR, Sauer BC, LaFleur J. Factors associated with screening or treatment initiation among male United States Veterans at risk for osteoporosis fracture. Bone. 2012;50(4):983-988.
21. Wu C-H, Chen C-H, Chen P-H, et al. Identifying characteristics of an effective fracture liaison service: systematic literature review. Osteoporosis Int. 2018;29(5): 1023-1047.

22. Wu C-H, Tu S-T, Chang Y-F, et al. Fracture liaison services improve outcomes of patients with osteoporosis-related fractures: A systematic literature review and meta-analysis. Bone. 2018;111:92-100.

23. Nakayama A, Major G, Holliday E, Attia J, Bogduk N. Evidence of effectiveness of a fracture liaison service to reduce the re-fracture rate. Osteoporosis Int. 2016;27(3):873-879.

24. Barker KL, Toye F, Lowe CJM. A qualitative systematic review of patients' experience of osteoporosis using meta-ethnography. Arch Osteoporos. 2016;11(1):33.

25. Rothmann MJ, Jakobsen PR, Jensen CM, Hermann AP, Smith AC Clemensen J. Experiences of being diagnosed with osteoporosis: a metasynthesis. Arch Osteoporos. 2018;13(1):21.

26. Chou L, Shamdasani P, Briggs AM, et al. Systematic scoping review of patients' perceived needs of health services for osteoporosis. Osteoporosis Int. 2017;28(11):3077-3098.

27. Sandelowski M, Barroso J. Handbook for Synthesizing Qualitative Research. New York: Springer Publishing Company; 2007.

28. Bondas T, Hall EO. Challenges in approaching metasynthesis research. Qual Health Res. 2007;17(1):113-121.

29. Finfgeld DL. Metasynthesis: The state of the art—so far. Qual Health Res. 2003;13(7):893-904

30. Saini M, Shlonsky A. Systematic Synthesis of Qualitative Research. Oxford: Oxford University Press; 2012.

31. Tong A, Flemming $\mathbf{K}$, McInnes E, Oliver S, Craig J. Enhancing transparency in reporting the synthesis of qualitative research: ENTREQ. BMC Med Res Methodol. 2012;12(1):181.

32. DeJean D, Giacomini M, Simeonov D, Smith A. Finding qualitative research evidence for health technology assessment. Qual Health Res. 2016;26(10):1307-1317.

33. National Osteoporosis Foundation. NOF Background: Founding and Milestones. 2017. Available at: https://www.nof.org/about-us/nof-background/. Accessed July 3, 2018.

34. Critical Appraisal Skills Programme. CASP Qualitative Checklist. 2017. Available at: http://www.casp-uk.net/checklists. Accessed July 3, 2018

35. Beaton DE, Sujic R, McIloy Beaton K, Sale J, Elliot-Gibson V, Bogoch ER. Patient perceptions of the path to osteoporosis care following a fragility fracture. Qual Health Res. 2012;22(12):1647-1658.

36. Sale JE, Beaton DE, Sujic R, Bogoch ER. 'If it was osteoporosis, I would have really hurt myself.' Ambiguity about osteoporosis and osteoporosis care despite a screening programme to educate fragility fracture patients. J Eval Clin Pract. 2010;16(3):590-596.

37. Sale JE, Bogoch E, Hawker G, et al. Patient perceptions of provider barriers to post-fracture secondary prevention. Osteoporos Int. 2014;25(11):2581-2589.

38. Sale JEM, Cameron C, Hawker G, et al. Strategies used by an osteoporosis patient group to navigate for bone health care after a fracture. Arch Orthop Trauma Surg. 2014;134:229-235.

39. Sale JEM, Hawker G, Cameron C, et al. Perceived messages about bone health after a fracture are not consistent across healthcare providers. Rheumatol Int. 2015;35(1):97-103.

40. Meadows LM, Mrkonjic L, Lagendyk L. Women's perceptions of future risk after low-energy fractures at midlife. Ann Fam Med. 2005;3(1):64-69.

41. Meadows LM, Mrkonjic LA. Breaking-bad news: women's experiences of fractures at midlife. Can J Public Health. 2003;94(6):427-430.

42. Meadows LM, Mrkonjic LA, Lagendyk LE, Petersen KM. After the fall: women's views of fractures in relation to bone health at midlife. Women Health. 2004;39(2):47-62.

43. Edwards BJ, Iris M, Ferkel E, Feinglass J. Postmenopausal women with minimal trauma fractures are unapprised of the existence of low bone mass or osteoporosis. Maturitas. 2006;53(3):260-266.

44. Feldstein AC, Schneider J, Smith DH, et al. Harnessing stakeholder perspectives to improve the care of osteoporosis after a fracture. Osteoporos Int. 2008;19(11):1527-1540.

45. Solimeo SL, Weber TJ, Gold DT. Older men's explanatory model for osteoporosis. Gerontologist. 2011;51(4):530-539.

46. Emmett CL, Redmond NM, Peters TJ, et al. Acceptability of screening to prevent osteoporotic fractures: a qualitative study with older women. Fam Pract. 2012;29(2):235-242.

47. Rothmann MJ, Huniche L, Ammentorp J, Barkmann R, Gluer CC Hermann AP. Women's perspectives and experiences on screening for osteoporosis (Risk-stratified Osteoporosis Strategy Evaluation, ROSE). Arch Osteoporos. 2014;9:192. 
48. Dreinhöfer KE, Anderson M, Féron J-M, et al. Multinational survey of osteoporotic fracture management. Osteoporosis Int. 2005; 16(2):S44-S53.

49. Mendis AS, Ganda K, Seibel MJ. Barriers to secondary fracture prevention in primary care. Osteoporosis Int. 2017;28(10):2913-2919.

50. Eisman J, Clapham S, Kehoe L. Osteoporosis prevalence and levels of treatment in primary care: The Australian BoneCare Study. J Bone Miner Res. 2004;19(12):1969-1975.

51. Solomon DH, Polinski JM, Stedman M, et al. Improving care of patients at-risk for osteoporosis: a randomized controlled trial. J Gen Intern Med. 2007;22(3):362-367.
52. Boudreau DM, Yu O, Balasubramanian A, et al. A survey of women's awareness of and reasons for lack of postfracture osteoporotic care. J Am Geriatr Soc. 2017;65(8): 1829-1835.

53. Weston JM, Norris EV, Clark EM. The invisible disease: making sense of an osteoporosis diagnosis in older age. Qual Health Res. 2011;21(12):1692-1704.

54. Elliot-Gibson V, Bogoch ER, Jamal SA, Beaton DE. Practice patterns in the diagnosis and treatment of osteoporosis after a fragility fracture: a systematic review. Osteoporosis Int. 2004;15(10):767-778. 